\title{
Silicon PV module customization using laser technology for new BIPV applications
}

\author{
Juan José García-Ballesteros ${ }^{1}$, Sara Lauzurica ${ }^{1}$, Miguel Morales ${ }^{1}$, Teodosio del Caño ${ }^{2}$, Daniel \\ Valencia $^{2}$, Leonardo Casado ${ }^{2}$, José Lorenzo Balenzategui ${ }^{3}$, Carlos Molpeceres ${ }^{1}$, \\ ${ }^{1}$ Centro Láser UPM, Universidad Politécnica de Madrid, \\ Ctra. de Valencia Km 7.3. 28031, Madrid, Spain. \\ ${ }^{2}$ Onyx Solar, C/ Rio Cea 1-46 05004 Ávila, Spain. \\ ${ }^{3}$ División de Energías Renovables, Energía Solar Fotovoltaica, \\ CIEMAT, Avda. Complutense, 22, 28040, Madrid, Spain
}

\begin{abstract}
It is well known that lasers have helped to increase efficiency and to reduce production costs in the photovoltaic (PV) sector in the last two decades, appearing in most cases as the ideal tool to solve some of the critical bottlenecks of production both in thin film (TF) and crystalline silicon (c-Si) technologies. The accumulated experience in these fields has brought as a consequence the possibility of using laser technology to produce new Building Integrated Photovoltaics (BIPV) products with a high degree of customization. However, to produce efficiently these personalized products it is necessary the development of optimized laser processes able to transform standard products in customized items oriented to the BIPV market. In particular, the production of semitransparencies and/or freeform geometries in TF a-Si modules and standard c-Si modules is an application of great interest in this market.

In this work we present results of customization of both TF a-Si modules and standard monocrystalline (m-Si) and policrystalline silicon ( $\mathrm{pc}-\mathrm{Si}$ ) modules using laser ablation and laser cutting processes. A discussion about the laser processes parameterization to guarantee the functionality of the device is included. Finally some examples of final devices are presented with a full discussion of the process approach used in their fabrication.
\end{abstract}

Keywords: Building Integrated Photovoltaics, Laser ablation; monolithic interconnection; amorphous silicon PV modules; crystalline silicon solar cells

\section{INTRODUCTION}

The use of renewable energy into architectural design is a field of the greatest importance to fulfil the present and future requirements of reduction of fossil fuels consumption. Under this scenario, an undeniable opportunity of developing and optimizing existing low carbon technologies emerges. Until now, PV technologies have been focused in the development of solar farms that do not take all the advantages and possibilities of this renewable source, and in most cases has been treated as a financial product due to the high incentives. Currently, big energy markets like Europe or USA require other business models. Nowadays, in the case of PV, the efforts should be focused in on-site energy generation as DER (distributed energy resource). In other words to bring the energy where it is demanded: the buildings. In this sense, the first approach has been carried out by means of BAPV (Building Applied Photovoltaics) leading critical main limitations: a) it requires an additional investment within the building budget, b) aesthetical does not fulfil the requirements of most of the building stakeholders showing poor acceptability and c) does not comply with key building codes limiting its application. Therefore, it is easy to understand that BIPV has the chance to become the most attractive market for PV technologies. This is based on the capacity of providing multifunctional solutions to buildings throughout active and passive properties allowing on-site RES (Renewable Energy Sources) energy production, thermal envelope benefits and daylighting entrance at the same time. At present, BIPV systems still represent a small share of the PV market mainly due to the mistaken belief that BIPV integrations are more expensive than conventional roof installations and that the strong incentives given in the past were associated only with "utility" products and not-distributed

Laser Processing and Fabrication for Solar, Displays, and Optoelectronic Devices III, edited by Edward W. Reutzel, Proc. of SPIE Vol. 9180, 91800H · C 2014 SPIE · CCC code: 0277-786X/14/\$18 · doi: 10.1117/12.2064084 
generation. Under this scenario, it is mandatory to carry out an effort in developing and breaking into the market of multifunctional BIPV solutions capable to satisfy the demands of main stakeholders in building sector in a holistic manner (cost, aesthetic, technical and structural needs) taking into account the benefits associated to the building users. In this context laser technology appears as key enable technology to fulfil two of the cited requirements: cost and aesthetic [1-5].

In fact lasers have been used in microelectronic and photovoltaic (PV) industry for decades. More recently, laser technology has appeared as a key enabling technology to improve efficiency and to reduce production costs. This tool is present not only in standard c-Si PV factories but it is becoming a fundamental technology to develop new concepts both in HE (High Efficiency) solar cells fabrication and in most of the TF (Thin film) photovoltaic technologies [6]. In addition laser technology could be a fundamental tool to solve a key problem in BIPV: the customization of standard PV devices, and in particular those based in silicon technologies, to fulfil the cost and aesthetic criteria imposed by the architectural design.

In the case of standard m-Si or pc-Si modules, a way to customize the module is doing free form geometries to change the standard appearance of the solar cells. With an appropriate cutting of the cell semitransparent modules or specific visual effects can be achieved, offering a device more appropriate to use in BIPV applications. The problem in this case is to produce a cutting process of outstanding quality (the process is made in a fully finished cell) to maintain as much as possible the characteristics of the device. Although some hybrid laser process like water jet laser guided systems are well suited to do that [7], we show in this work that even with standard Diode Pumped Solid State Lasers (DPSS) in the ns regime it is possible to achieve outstanding results with standard laser cutting processes. As we will show in the study, in this kind of approach it is very important to preserve not only the electrical performance of the device, but the mechanical performance as well, in order to be able of finish the module using standard encapsulation processes.

For TF a-Si modules, the technology of semitransparent modules using laser technology has been developed at industrial level several years ago [8]. In fact the production of a standard TF a-Si module on glass in superstrate configuration implies a number of laser ablation steps to achieve the monolithical interconnection of the device. The three laser process needed are usually named P1, P2 and P3 (Fig.1). P1 is intended to remove locally the TCO (Transparent Conductive Oxide) used as front contact in order to define the individual cells. In the P2 process, a laser irradiation through the glass ablates the PV absorbent structure (single junction p-i-n a-Si structure) and finally the P3 removes both the PV absorbent and the aluminium back contact, interconnecting the individual cells. In addition P4 processes, in which the full structure of front contact, absorbent and back contact is ablated, can be done for edge deletion or isolation processes.

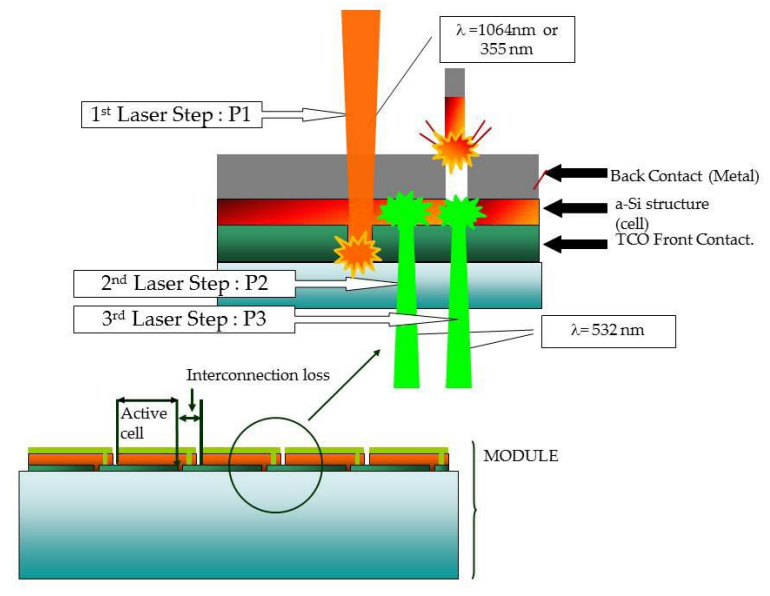

Figure 1. Laser ablation processes for monolithic interconnection of single junction a-Si Thin Film PV modules

Optimized P3 or even P4 processes can be used to produce customized semitransparent modules in which the transparency degree is controlled by means of the number of laser lines that are produced in addition of those needed to interconnect the module. However the accumulation of laser grooves in the devices decrease the performance in the device, limiting in commercial semitransparent modules the degree of transparency to values around $30 \%$. In this work we discuss the possibility of using different approaches based in laser technology not only to produce semitransparent 
modules but as well to produce customized images in the device which could improve the use of this elements in BIPV applications.

\section{EXPERIMENTAL}

\subsection{Samples description}

For experiments of customization of standard PV silicon modules we have used monocrystalline (m-Si) and polycrystalline (pc-Si) commercial silicon wafers. Thickness of both type of cells were $250 \mu \mathrm{m}$.

For TF module customization we worked with two different kind of samples. First we parameterized the process using $10 \mathrm{~mm} \times 10 \mathrm{~mm}$ TF single junction a-Si minimodules with aluminium back contact (layers structure is described in Table II). In addition fully commercial modules $(1200 \mathrm{~mm} \times 1200 \mathrm{~mm})$ with the same structure and without encapsulation were used for final customization processes.

Table 1. Structure of the a-Si modules used in the study.

\begin{tabular}{lcr} 
Layer & Material & Thickness $(\mathbf{n m})$ \\
\hline Front contact & Asahi-U (SnO2:F) & 800 \\
absorbent & $p-i-n$ a-Si & 300 \\
Back Contact & Aluminium & 150
\end{tabular}

\subsection{Laser sources and irradiation set-up}

For cell cutting in c-Si cells we used different DPSS working in at 355 and $1064 \mathrm{~nm}$. To maintain the best performance in source stability, pulse energy is controlled for every wavelength with an external energy attenuator. The optical set-up includes a galvanometric scanner or fix optics with the characteristics summarized in Table 2.

Table 2. Characteristics of the lasers and irradiation systems used in c-Si cells cutting studies for module customization

\begin{tabular}{lcccc}
\hline Laser and Optics & $\boldsymbol{\lambda}(\mathbf{n m})$ & $\begin{array}{c}\text { Pulse } \\
\text { width }(\mathbf{n s})\end{array}$ & $\begin{array}{c}\text { Spot Size in sample } \\
(\boldsymbol{\mu} \mathbf{m})\end{array}$ & Scanner field \\
\hline $\begin{array}{l}\text { Spectra Physics X15 Navigator } \\
+ \text { Ray Laser SuperScan 2 }\end{array}$ & 1064 & 15 & 45 & $150 \mathrm{~mm} \times 150 \mathrm{~mm}$ \\
$\begin{array}{l}\text { Spectra Physics Hippo } \\
+ \text { ScanLab HurryScan }\end{array}$ & 355 & 15 & 30 & $120 \mathrm{~mm} \times 120 \mathrm{~mm}$ \\
$\begin{array}{l}\text { Spectra Physics Pulseo } \\
+ \text { fix optics }\end{array}$ & 355 & 38 & 28 & - \\
\hline
\end{tabular}

For TF ablation studies in TF module customization we used DPSS lasers emitting at $532 \mathrm{~nm}$ and $1064 \mathrm{~nm}$ according to the characteristics described in Table 3.

Table 3. Characteristics of the lasers and irradiation systems used in TF ablation studies for module customization

\begin{tabular}{lcccc}
\hline Laser and Optics & $\lambda(\mathbf{n m})$ & $\begin{array}{c}\text { Pulse } \\
\text { width }(\mathbf{n s})\end{array}$ & $\begin{array}{c}\text { Spot Size in sample } \\
(\boldsymbol{\mu} \mathbf{m})\end{array}$ & Scanner field \\
\hline $\begin{array}{l}\text { Spectra Physics Explorer } \\
+ \text { ScanLab HurryScan }\end{array}$ & 532 & 15 & $25-48$ & $150 \mathrm{~mm} \times 150 \mathrm{~mm}$ \\
$\begin{array}{l}\text { Powerlaser Naos i20-M } \\
+ \text { Raylase AXIALSCAN-30-Y }\end{array}$ & 1064 & 50 & $50-80$ & $600 \mathrm{~mm} \times 600 \mathrm{~mm}$ \\
\hline
\end{tabular}




\subsection{Sample Characterization}

The quality of the cutting kerfs and the laser scribes were assessed using optical microscopy (Olympus PMG3) and confocal laser microscopy (LeicaDCM3D). In addition, SEM images are included for a better description of the morphology and to obtain qualitative information about the ablation processes.

In order to study the effect of the laser processes in the performance of the solar cells, the current-voltage characteristics $(\mathrm{J}-\mathrm{V})$ were measured in dark and under calibrated $100 \mathrm{~mW} / \mathrm{cm}^{2}$ AM1.5G irradiance.

Finally, in the study of cutting processes in c-Si solar cells, we include some measurements of electroluminescence to discuss the effects of microcracks formation under certain circumstances. The equipment used was a pco.1300 Solar with a resolution of 1392 x 1040 pixel and a quantum efficiency up to 11\% @ 900nm.

\section{RESULTS AND DISCUSSION}

\subsection{Customization by laser cutting of silicon solar cells}

This study was focus on obtaining square cut geometries for semitransparent c-Si module fabrication using standard laser cutting processes. A number of semitransparent test modules using both commercial $\mathrm{m}-\mathrm{Si}$ and pc-Si solar cells were fabricated. The selection of the geometry was based only in aesthetic criteria, which is a fundamental aspect in BIPV applications.

In a first round of experiments, we optimized the cutting quality of a single square of $50 \mathrm{~mm} \times 50 \mathrm{~mm}$ centered in the cell. We assessed the cutting process at $1064 \mathrm{~nm}$ and $355 \mathrm{~nm}$. For IR we used a galvanometric scanner to guide the beam to the workpice. On the other hand, for $355 \mathrm{~nm}$ we assessed the process using both scanner irradiation and fix optics according to the information provided in Table 2.

Excellent cutting conditions were found both for $\mathrm{m}-\mathrm{Si}$ and pc-Si cells. Table 4 shows the process parameters used in the best cutting conditions found in this study for cutting the cells defined in section 2.1. In all cases multiple pass with the beam over the workpiece is necessary to achieve cutting of the whole cell thickness. The optimum process parameters were selected according to the electrical behaviour of the cells after the cutting process both under dark and illumination conditions. We used the same parameters for cutting $\mathrm{m}$-Si and pc-Si cells (the thickness of the cells was the same) and we observed similar cell degradation in both cases due to the cutting process.

Table 4. Laser cutting parameters

\begin{tabular}{lccccc}
\hline $\boldsymbol{\lambda}(\mathbf{n m})$ & $\begin{array}{c}\text { Rep. } \\
\text { Rate }(\mathbf{H z})\end{array}$ & $\begin{array}{c}\text { Spot Size } \\
(\boldsymbol{\mu} \mathbf{m})\end{array}$ & $\begin{array}{c}\text { Pulse Energy } \\
(\boldsymbol{\mu} \mathbf{J})\end{array}$ & $\begin{array}{c}\text { Process Speed } \\
(\mathbf{m m} / \mathbf{s})\end{array}$ & $\begin{array}{c}\text { Number of } \\
\text { Repetitions }\end{array}$ \\
\hline 1064 & 20000 & 45 & 235 & 6 & 7 \\
355 (scanner) & 50000 & 28 & 90 & 30 & 25 \\
355 (fix opt.) & 50000 & 30 & 200 & 3 & 3 \\
\hline
\end{tabular}

As an example of the results obtained, Fig. 3 (left) shows the variation of the cell characteristics under illumination of a reference untreated cell and other treated cut with optimum laser parameters for the different conditions studied. The shape of the curve indicates an extremely good cutting quality. Fig. 3 (right) shows a SEM image of the cutting kerf taken in an area that contains a metallization finger. The morphology of the cutting kerf can be considered appropriate, with limited thermal affection in the processed section of the workpiece. 

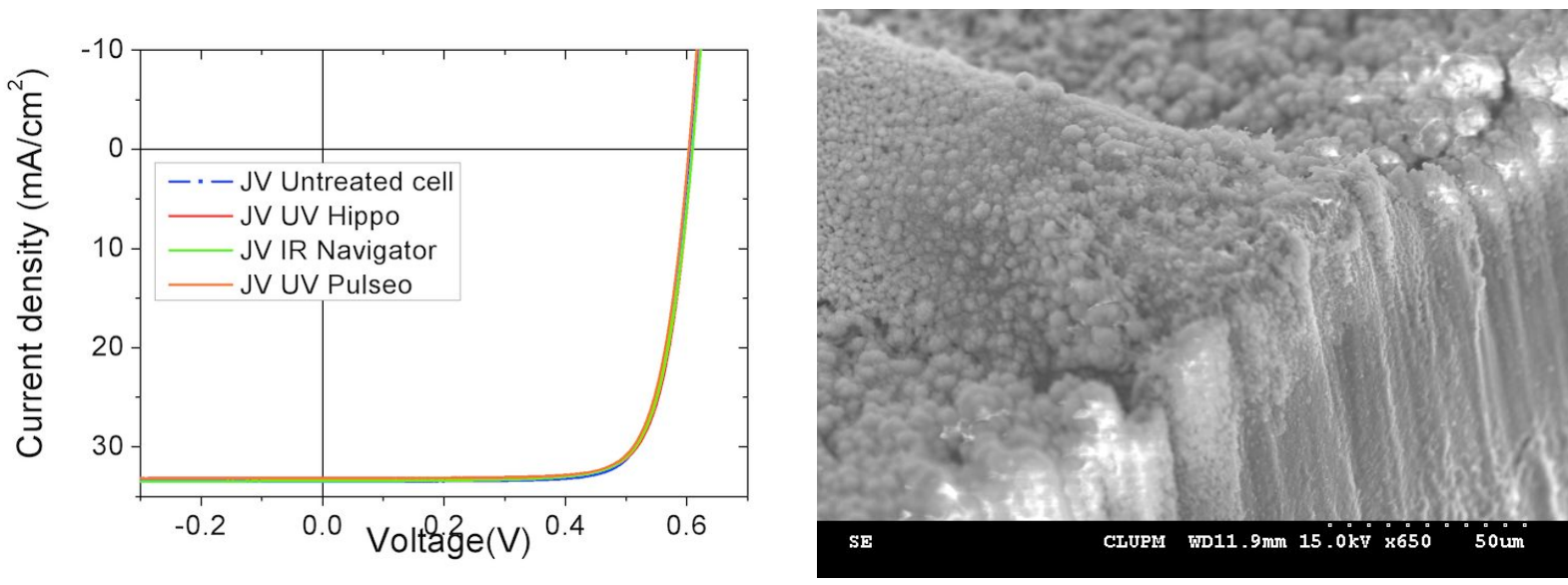

Fig. 2 Left: Reference characteristic curve for an untreated cell and corresponding curves for cut cells with different lasers. Right: SEM image of the cutting kerf of the cell in a section coincident with a metallization finger.

Although the results shown before corresponding to measurements under illumination shown a very similar behaviour, dark current-voltage measurements shown a slightly better behaviour for the cutting processes done at $355 \mathrm{~nm}$ with galvanometric scanner. For that reason we selected this approach as the reference process and the results that we are going to discuss from now on were obtained with cutting processes performed under these process conditions.

We continued our study assessing the effect of the geometry itself on the cell performance. We defined four geometries of interest (Fig. 4) and we studied the influence of the cutting process in the characteristic of the cells. For CUT 1 and CUT 2 geometries the processes shown a high degree of repeatability, however for CUT3 and CUT4 the dispersion in the electrical parameters of the cell presented a slightly higher dispersion. In fig 4 we present results for typical performance of CUT1-CUT4 cells. For CUT1 and CUT2 we present values for a single cell (the values obtained for different cells present almost no dispersion). On the other hand for CUT3 and CUT4 geometries we include values corresponding to two different cells to demonstrate that to increase the number of cut areas inside the cell leads to a less reproducible process.

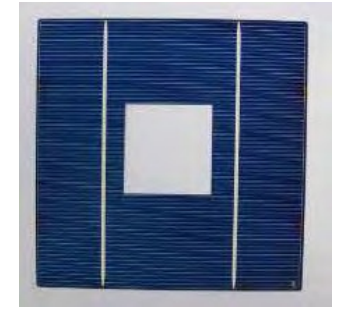

CUT 1

Square size $50 \mathrm{~mm} \times 50 \mathrm{~mm}$



CUT 2

Rectangle size $96 \mathrm{~mm} \times 50 \mathrm{~mm}$



CUT 3

Squares size $11 \mathrm{~mm}$ x $11 \mathrm{~mm}$

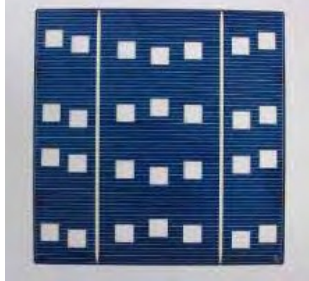

CUT 4

Squares size $11 \mathrm{~mm} \times 11 \mathrm{~mm}$

Fig 3. Different cutting geometries considered in this study

The results obtained show two main reasons for the power losses in the processed cells. The first is related with the obvious fact that after the cutting process less active area remains in the cut cells (unavoidable in any case). The second effect is a reduction of the Fill Factor. This second effect (Fig. 4) is greatly dependent on the cutting geometry. The Fill Factor degradation is mainly due to a higher series resistance value, probably related with the interruptions in the metallization grid imposed by the cutting process. We didn't found significant changes in the shunt resistance values, a clear indication that the cutting processes is of outstanding quality in terms of shunting formation and bulk material damaging. 

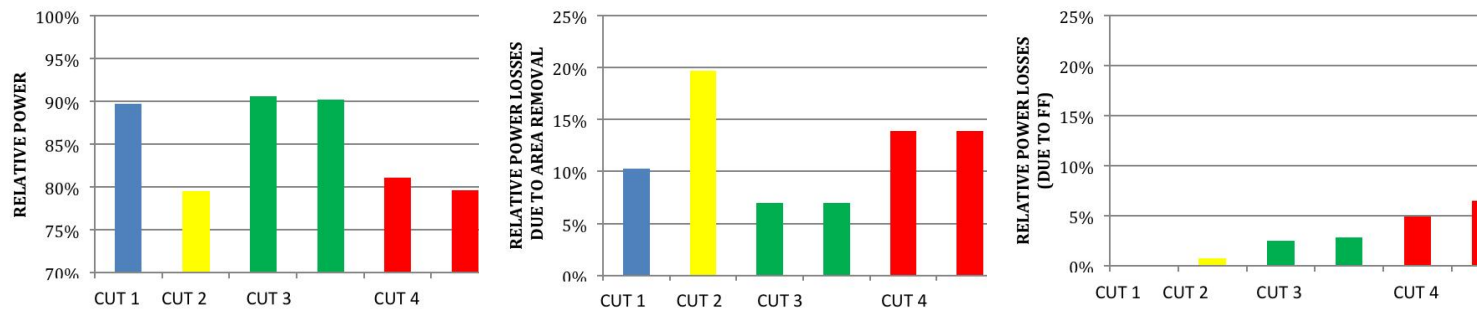

Fig 4. Analysis of the relative power (left) obtained with the different cut geometries. Relative power losses associated to area removal (center) and relative power losses due to Fill Factor degradation (right) indicates an outstanding quality of the laser cutting process.

After this study we select CUT 1 geometry to finish a complete module. The module was done cutting squares of $50 \mathrm{~mm}$ x $50 \mathrm{~mm}$ in single cells and then finishing the module with standard soldering and encapsulation processes. In each module we include 40 cells. To create the desired aesthetic effect we displace the cut square some millimeters between adjacent cells. The final effect is a partially transparent module with the desired aesthetic effect of undulatory geometry. Fig shows the final module and the module parameters obtained with a calibrated flash tester equipment.

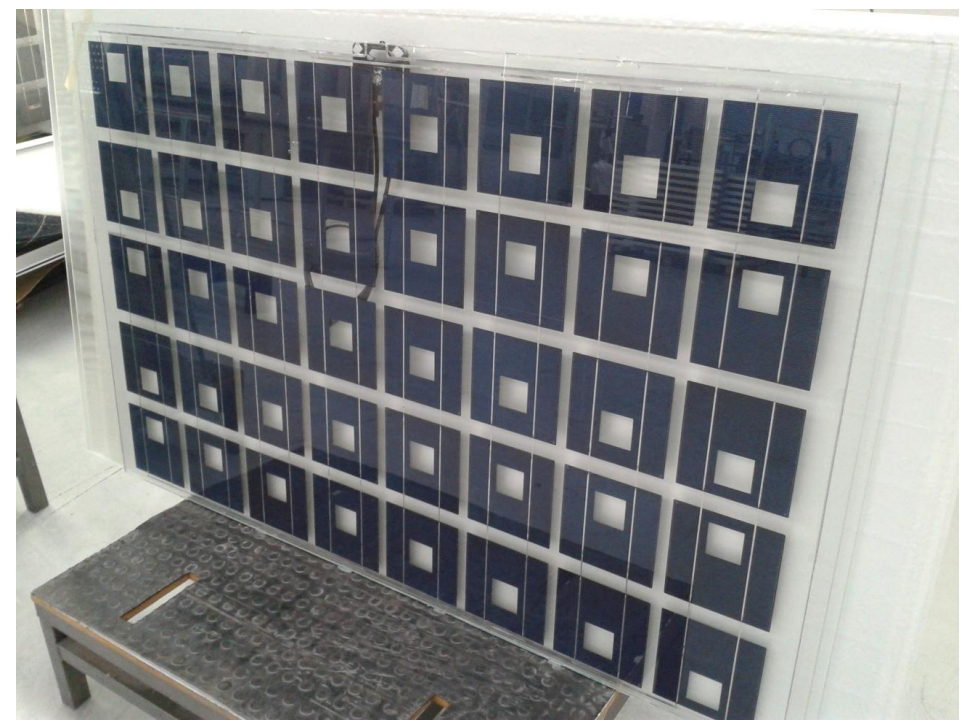

MODULE DATA

$\mathrm{I}_{\mathrm{sc}} / \mathrm{I}_{\mathrm{mp}}[\mathrm{A}]=7.26 / 6.70$
$\mathrm{Voc} / \mathrm{Vmp}[\mathrm{V}]=25.62 / 19.16$
F.F. $=0.718$

Cell data before/after cutting:

\begin{tabular}{lrr} 
& Before & After \\
\hline Isc & 7,23 & 7,18 \\
Voc & 0,61 & 0,60 \\
Pm & 3,38 & 3,33 \\
Im & 6,67 & 6,66 \\
Vm & 0,51 & 0,50 \\
FF & 76,71 & 76,96
\end{tabular}

Fig. 5. Picture of the finished semitransparent module and performance data obtained with a flash tester. Data provided for module has been measure using an standard 6" p-Si module as reference. The table shows data for one of the cells before and after the cutting process.

This module was finished with a glass $(4 \mathrm{~mm}+4 \mathrm{~mm}) /$ EVA encapsulation with soldering processes manually done. In fact automatic soldering led to the rupture of a significant number of cells due to the weakening induced by the cut geometry in the mechanical performance of the cells. In order to minimize the number of cells rejected or broken during the soldering and encapsulation process we tried to optimize the cutting geometry avoiding sharp corners in the cut squares. Microcracks formation, and then a reduction of the mechanical performance of the cell, is produced more easily during the cutting process when very small curvature radius is introduced in the cutting geometry. As an example Fig. 6 shows a electroluminescence image of a cell cut with sharp corners in the square. A microcrack is clearly visible in the top-left corner of the depicted cell. These microcracks has low influence in the electrical performance of the cell, but have a great influence in its mechanical performance. We minimized this problem changing the geometry of the square 
corners to introduce a higher curvature radius for the cutting tool (see please Fig. 6). Only introducing this change we could decrease substantially the number of broken cells during the back-end process.
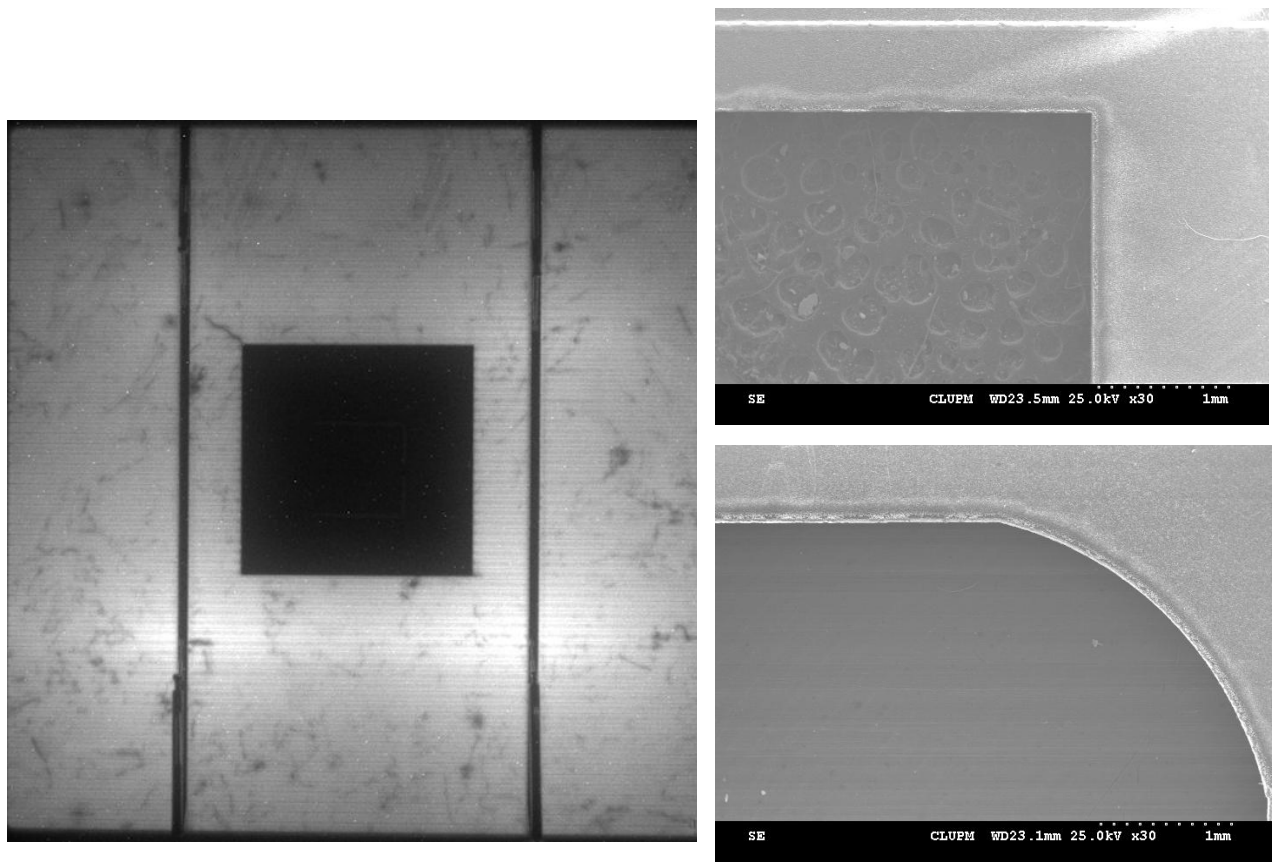

Fig. 6. Left: Electroluminiscence image of a p-Si cut solar cell with sharp corners in the cut square. A microcrack, generated by the thermomechanical effects associated to the cutting process, is clearly visible in the top-left corner. Right: Original sharp corner geometry (top) and modified corner geometry with a higher curvature radius (bottom)

Finally we must highlight that the module parameters are appropriate for a BIPV product, fulfilling fundamentally the requirements of aesthetical criteria. In addition, and bearing in mind that the processes imply only fully commercial DPSS lasers in the ns range, we think that this approach offers the possibility of customize standard c-Si PV modules fabrication with clear cost advantages if compare with alternative technologies.

\subsection{Customization by laser ablation of TF a-Si modules}

In this second approach we studied the possibility of customize TF a-Si PV modules in superstrate configuration. In this kind of PV devices, total or partial elimination of the layers forming the module lead to semitransparency effects that can be used to produce BIPV products. This effect is well known in PV industry and semitransparent modules made with laser technology are a standard product in the market.

In practice, a very effective way to remove totally or selectively these layers is using laser ablation process. P3 or P4 ablation steps can be used to produce lines along the module, being the final result slightly different due to the diffusive effect produce by the transparent conductive oxide used as front contact if P3 processes are considered. In any case the density of ablation lines determines the transparency degree in the module.

Taking into account that accumulation of laser processes degrade the module performance due to shunting generation [9], and that high degrees of transparency imply an important quantity of active area removal, usually low values of transparency (around $10 \%$ ) are commercially available. 
In this context, our work presents another interesting approach for BIPV products. The idea is to produce free-form geometries inside the active area of the module to generate more complex aesthetic effects than simple transparencies. For that we need to remove both the absorbent structure and the back contact (like in a P3 process) or the full structure including the front contact (like in a $\mathrm{P} 4$ process).

The problem is that introducing complex geometries using P3 or P4 processes starting and ending inside the module easily degrade the device characteristics. Even with a extremely good control of the irradiation system and quite sophisticated optoelectronic instrumentation, to minimize effects of pulse overlapping due the acceleration and brake down of the positioning system (linear axis or galvanometric scanners), to avoid a considerable damage in the device is difficult. The process parametric window is usually very narrow and the degradation of the module performance is unavoidable (Fig. 7).
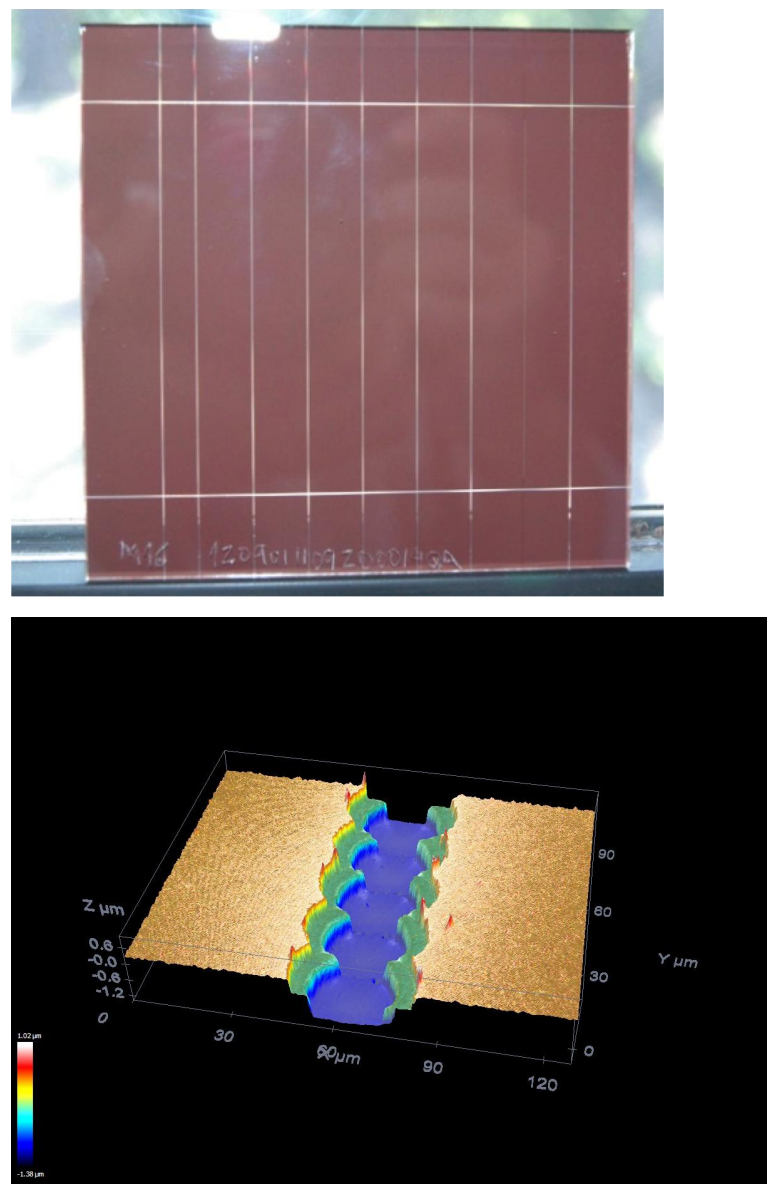


Fig. 7 Left-top: Minimodule used for P3/P4 process optimization.. A complete scribe process is made parallel to the interconnection lines in one of the active cells to study the damage induced in the device by the process. Right-top: Comparison of the module performance when optimized and unoptimized processes are introduced in one of the cells. Left-Bottom: Confocal image of an optimized P4 process. Left-Bottom: Comparison of the characteristic curve of an active cell unprocessed with an optimized P4 process.

The approach followed in this work is to define the geometry to be introduced in the module ablating the PV structure until the front contact or until the glass substrate. Then we perform an electrical isolation of the treated area using optimized $\mathrm{P} 4$ processes. The advantage of this approach is that the ablation processes in the region in which the required geometry must be defined (what we usually name P3 and P4 unoptimized processes), can be done with a higher throughput. The reason is that even if the processes introduce shunting of the device, the posterior isolation of the treated area guarantees the appropriate performance of the remaining area of the PV module. 
For this study we started defining optimal P3 and P4 processing conditions for the minimodules described in Section 3.1. Figure 8 shows confocal microscopy profiles of optimum P3 and P4 processes. Laser irradiation parameters for these optimal conditions are included.
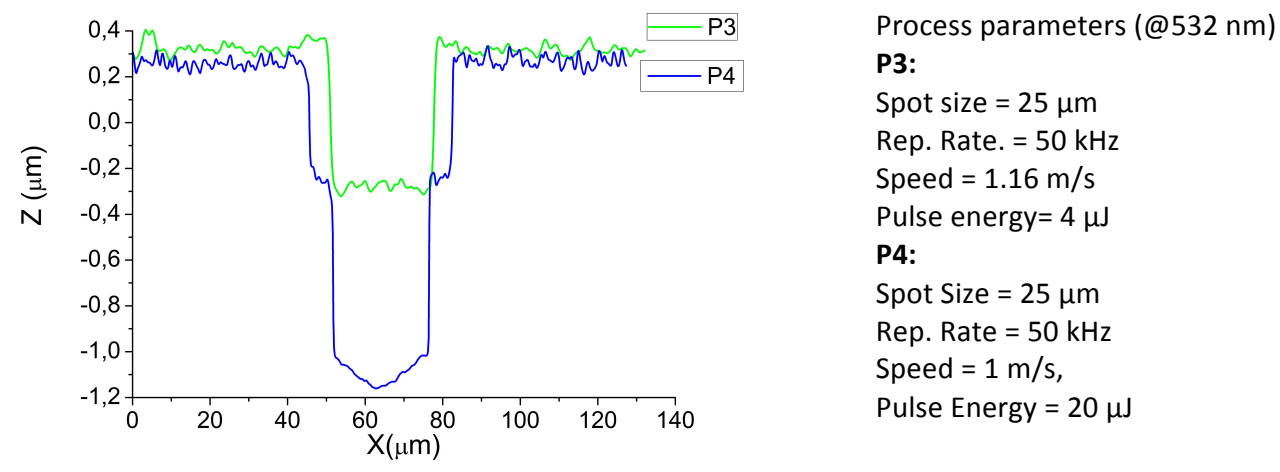

Fig. 8 Profiles for optimized P3 and P4 processes obtained with confocal microscopy. The edge of the grooves in any part of the structure is very clean and shows no signs of thermal affection.

As an example of the results achieved, Fig. 9shows a company logo scribed in a minimodule of $10 \mathrm{~mm} \times 10 \mathrm{~mm}$. The logo has been made using P4 unoptimazed process (all structure is removed but it is not necessary to avoid shunting of the device, so a higher throughput can be achieved). In addition we isolated the area occupied by the image using two additional optimized P4 isolation processes. These P4 are made perpendicular to the interconnection P1, P2 and P3 laser scribes. The original border isolation process made by the module supplier and the two P4 scribes made for logo isolation are identified in the image. Characteristic curves for the complete module and for each active area after the logo isolation are shown. The fact that the total intensity is exactly the addition of that corresponding to the individual active areas indicates and outstanding quality of the P4 processes.
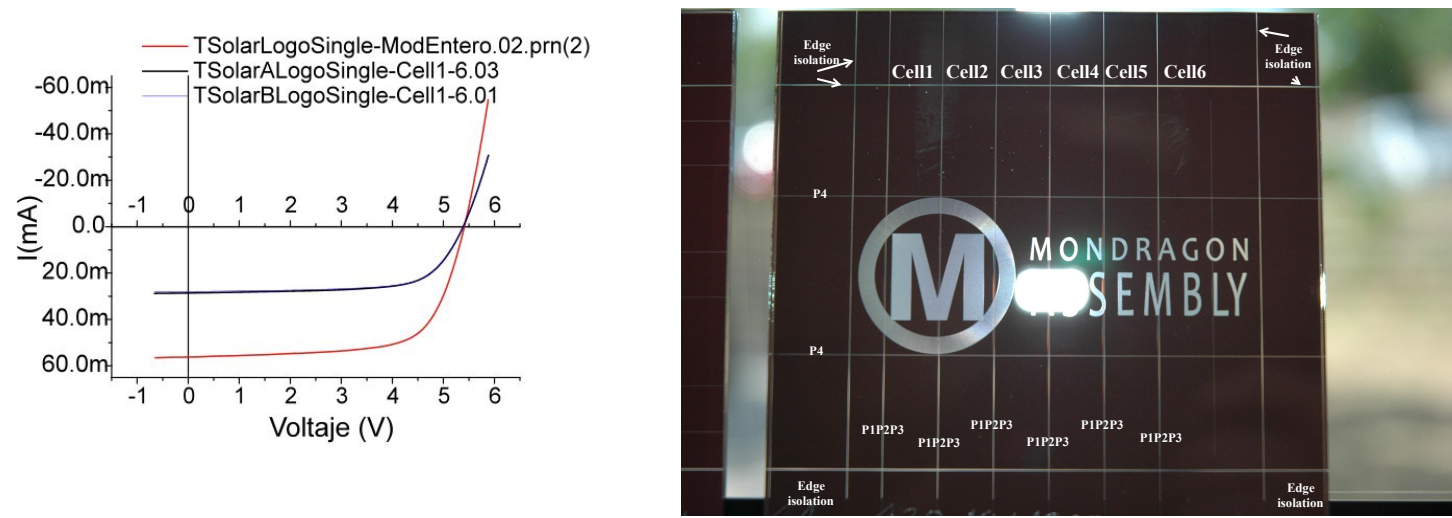

Fig. 9: Left: Characteristic curves for the complete module and for each individual part after image scribing. Right: Final result of a company logo inscribed in an active $10 \mathrm{~mm}$ x $10 \mathrm{~mm}$. The area in which the logo is inscribed is isolated by means of P4 optimizied processes.

Finally we applied this approach to full size a-Si PV modules of $1200 \mathrm{~mm}$ x $1200 \mathrm{~mm}$. New generation of digitally controlled 3D galvanometric scanners allows big area processing (up to $2000 \mathrm{~mm}$ x $2000 \mathrm{mmm}$ ). In our study we used a $600 \mathrm{~mm}$ by $600 \mathrm{~mm}$ scanning field to produce a semitransparent company logo using P3 processes with an effect of semitransparency $(20 \%)$ in the area defined by the logo. To achieve a higher throughput we scribed the module with an IR laser according to the data provided in Table 3. After logo processing we isolated the damaged area using optimized 
P4 scribes with the conditions described in Fig. 8. The result (Figure 10) is a customized a-Si module fully compatible with BIPV applications.

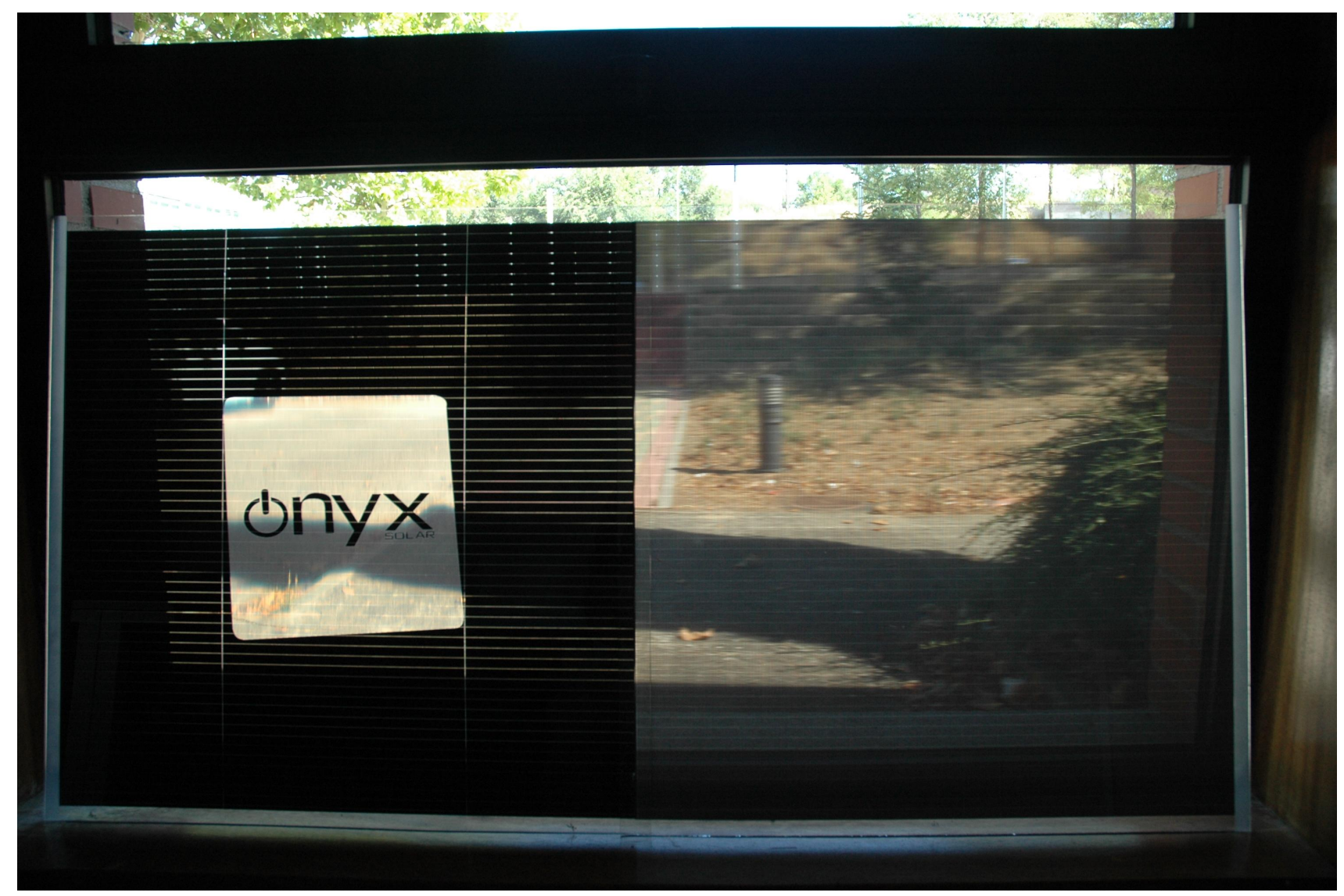

Fig. 10: Customized module with the Onyx logo showing semitransparency effects (left). For comparison an standard semitransparent $(20 \%)$ a-Si module of the same size is shown (right).

\section{CONCLUSIONS}

BIPV products will be one the more attractive areas for PV industry in the next years. In particular, customized semitransparent modules are one of the aesthetical effects that BIPV industry consider as standard product to be integrated in their portfolio. In this work we have shown that laser technology is ideally suited to customize standard PV silicon products producing final functional devices with different transparency effects.

In standard c-Si technologies, both m-Si and pc-Si solar cells can be cut with standard commercial lasers in the ns range with outstanding results in cut quality, avoiding the use of expensive hybrid technologies.

In $\mathrm{TF}$ a-Si modules, laser selective ablation processes similar to those used for monolithic interconnection can be adapted for module customization. In particular complex geometries with semitransparency effects can be integrated in superstrate TF a-Si modules offering very attractive functional products from the aesthetical point of view, a key requirement in the field of BIPV. 


\section{ACKNOWLEDGEMENTS}

This work has been supported by the Spanish Ministry of Science and Innovation under projects AMIC ENE201021384-C04-02/04, and INNDISOL IPT-420000-2010-6 (FEDER funded "Una manera de hacer Europa").

\section{REFERENCES}

[1] EU Commission. "A European Strategic Energy Technology Plan (SET Plan)”. COM/2007/0723, 2007.

[2] Building Performance Institute Europe. "Europe's building under the microscope, 2011.

[3] EU Commission. "Energy Performance of Building Directive". Directive 2010/31/EU.

[4] EuroACe. "EuroAce position on the EU Efficiency Plan 2011".

[5] Jelle, B., Breivik, C., Røkenes, H.D., "Building integrated photovoltaic products: A state-of-the-art review and future research opportunities". Solar Energy Materials \& SolarCells 100 69-96 (2012)

[6] Booth, H., "Laser Processing in Industrial Solar Module Manufacturing". Journal of Laser Micro/Nanoengineering, 5(3), 183-191 (2010).

[7] Perrottet, D., Boillat, C., Amorosi, S., Richerzhagen, B. "PV processing: Improved PV-cell scribing using water jet guided laser" Refocus 6 (3), 36-37 (2005)

[8] Wang, J., Wang, H., Du, J. Sun, R. Xu, C., Zhang, Y., Wang, D., Liu, T. Huang, Y., Jia, H. Mai, Y. "Performance improvement of amorphous silicon see-through solar modules with high transparency by the multi-line ns-laser scribing technique" Optics and Lasers in Engineering 51 1206-1212 (2013)

[9] Garcia-Ballesteros, J.J., Torres, I., Lauzurica, S., Canteli, D., J.J.Gandia, J.J., Molpeceres, C. "Influence of laser scribing in the electrical properties of a-Si:H thin film photovoltaic modules" Solar Energy Materials \& Solar Cells 95 (3) 986-991 (2011) 